\title{
Obstructive sleep apnea and atrial fibrillation
}

\author{
This article was published in the following Dove Press journal: \\ Nature and Science of Sleep \\ 14 April 2010 \\ Number of times this article has been viewed
}

\section{Keith Todd \\ William F Mclntyre \\ Adrian Baranchuk \\ Department of Cardiology, Kingston General Hospital, Queen's University, Kingston, Ontario, Canada}

Correspondence: Adrian Baranchuk Assistant Professor of Medicine, Cardiac Electrophysiology and Pacing, Kingston General Hospital K7L 2V7, Queen's University, Kingston, Ontario, Canada $\mathrm{Tel}+$ I 6135496666 ext 380 I Fax + I 6I3548 I387 Email barancha@kgh.kari.net

\begin{abstract}
Atrial fibrillation (AF) is the most common sustained arrhythmia. The clinical impact of AF results primarily from its association with stroke, heart failure, and increased overall mortality. Recently there has been increasing evidence of an important association between obstructive sleep apnea (OSA) and AF. OSA is a common breathing disorder affecting an estimated 5\% of the population and is highly prevalent in patients with established cardiovascular disease. The prevalence of OSA among patients with AF is remarkable with estimates ranging from $32 \%-49 \%$. The pathophysiological connection between AF and OSA remains speculative, but appears to be the result of apnea-induced hypoxia, intrathoracic pressure shifts, inflammation, heightened sympathetic activity, and autonomic instability leading to hypertension, diastolic dysfunction, left atrial enlargement, and electrical remodeling. Initial results appear promising that intervention with continuous positive airway pressure may be effective in reducing the burden of $\mathrm{AF}$ in this population, however, further investigations are needed. Here, we review the literature on the current epidemiologic data, pathophysiology, and therapeutics linking these two common conditions.
\end{abstract}

Keywords: cardiac arrhythmia, autonomic imbalance, intrathoracic pressure changes, diastolic dysfunction, hypertension, sleep-disordered breathing

\section{Introduction}

Atrial fibrillation (AF) is the most common sustained arrhythmia encountered in the US, affecting an estimated 2.3 million people, and accounting for one third of all cardiac rhythm hospitalizations. ${ }^{1}$ It is estimated that individuals aged 40 years and older have a lifetime risk of one in four of developing $\mathrm{AF}^{2}$ and that 5.6 million people in the US will be affected by $2050 .^{3}$ The clinical impact of AF results primarily from its association with stroke, heart failure, and increased overall mortality. ${ }^{4,5}$ There are many diseases associated with increased risk of AF including hypertension, coronary artery disease (CAD), valvular heart disease, congestive heart failure (CHF), and obesity as well as several others. ${ }^{1}$ Recently, there has also been increasing evidence of an important association between obstructive sleep apnea (OSA) and AF. ${ }^{6}$

OSA is a common disorder affecting an estimated $5 \%{ }^{7}$ of the population, or approximately 15 million adults in the US, and is highly prevalent in patients with established cardiovascular disease. ${ }^{8,9}$ The prevalence of OSA among patients with $\mathrm{AF}$ is remarkable with estimates ranging from $32 \%-49 \%,{ }^{10}$ providing support for an association between the conditions. While studies performed thus far have provided some insight into the pathological interactions of these conditions, further investigation into this emerging association is required. submit your manuscript | www.dovepress.com

Dovepress 


\section{Methods}

We review literature on the current epidemiologic data, pathophysiology, and therapeutics linking these two common conditions. Articles were selected from a computerized literature search in the Medline database using the keywords: "sleep apnea syndromes", "sleep apnea", "obstructive sleep apnea", "sleep disorders", "snoring", "respiration disorders", "airway obstruction", "atrial fibrillation", "cardiac arrhythmias", "autonomic nervous system", and all the possible combinations of the above. Two independent investigators $(\mathrm{KT}, \mathrm{AB})$ reviewed the abstracts and selected the ones considered of interest for the review. Discrepancies were solved by consensus.

\section{OSA-pathophysiology}

OSA is defined by repetitive upper airway collapse that occurs during sleep producing an interruption of ventilation that results in subsequent hypoxia, hypercapnia, sleep arousals, shifts in intrathoracic pressure, and heightened sympathetic activity. ${ }^{9}$

This milieu of pathologic changes results in a sequence of events thought to promote cardiac and vascular disease.
The individual role of these and other pathologic changes and how they contribute to arrhythmogenesis and AF is outlined briefly below and illustrated in Figure 1.

\section{Intrathoracic pressure changes}

OSA results in repetitive inspiration against a collapsed upper airway that can generate substantial shifts in intrathoracic pressure, producing gradients approaching $-65 \mathrm{mmHg}{ }^{8}$ These gradients are then transmitted from the thorax to the thin-walled atria and the transmural forces are thought to contribute to atrial chamber enlargement and fibrosis, both known risk factors for AF. ${ }^{1,11}$ In addition, it has been suggested that these transmural forces may be important in tissue stretch and remodeling at the pulmonary vein (PV) ostia, ${ }^{11}$ a known focal source of AF. ${ }^{12}$

\section{Instability of autonomic tone/sympathetic activation}

Nocturnal apneas occur repeatedly during sleep in OSA patients and are accompanied by chemoreceptor induced sympathetic activation and/or decreased parasympathetic tone that has been demonstrated through impaired vagal input,

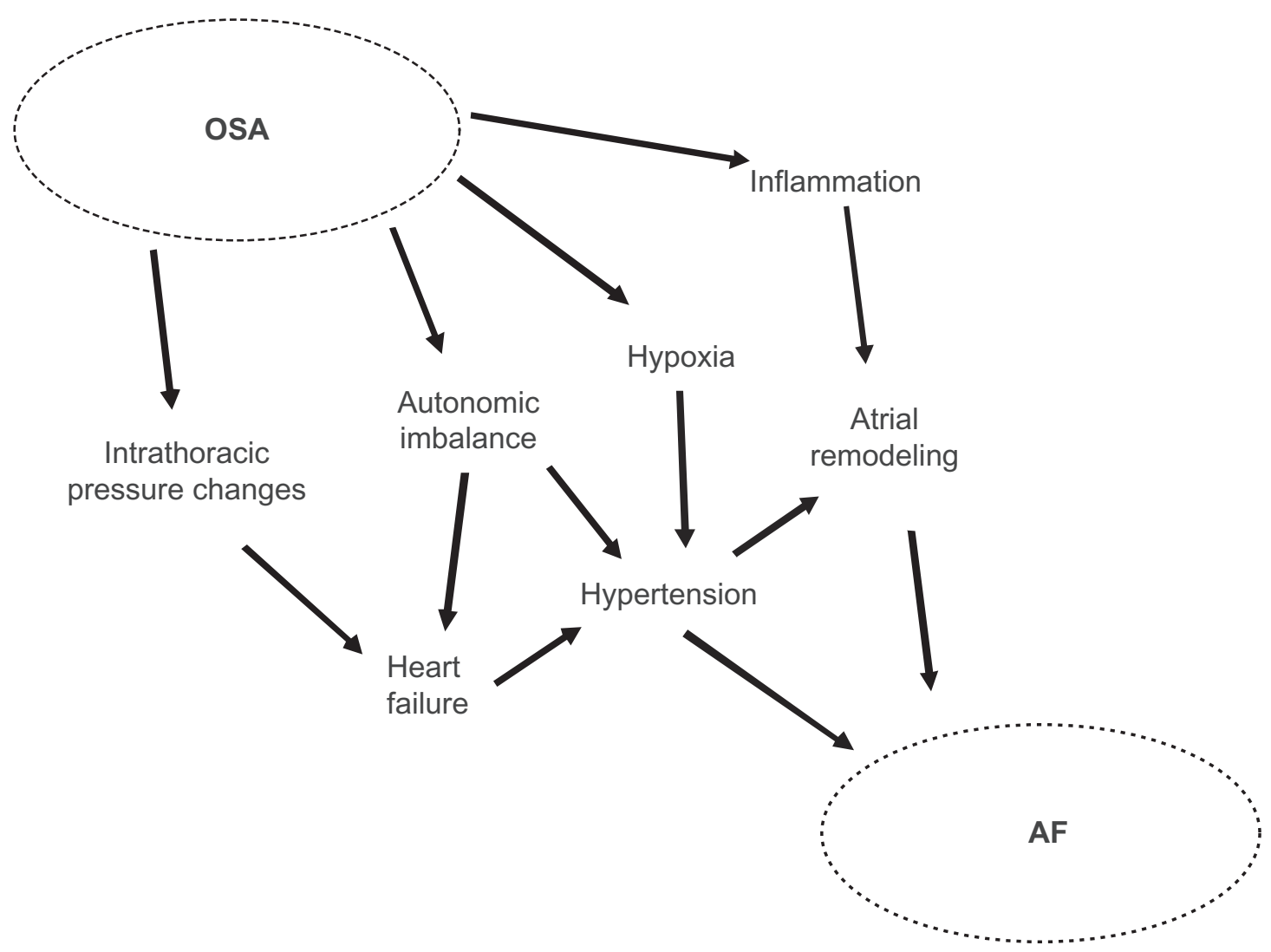

Figure I Diagram demonstrating the putative interactions of the pathophysiological mechanisms implicated in linking OSA and AF. Abbreviations: AF, atrial fibrillation; OSA, obstructive sleep apnea. 
diminished baroreflex sensitivity, and impairment of the parasympathetic components of heart rate variability. ${ }^{6}$ Decreased heart rate variability and increased blood pressure variability are known to elevate cardiovascular risk including heart failure, myocardial infarction, arrhythmic complications, and death, and have been associated with OSA in several studies. ${ }^{13,14}$

The mechanism by which this heightened sympathetic activity occurs has yet to be elucidated ${ }^{8}$ but has also been demonstrated as peripheral vasoconstriction resulting in markedly elevated systemic blood pressures. ${ }^{15,16}$ A strong association between OSA and hypertension has been reported extensively, ${ }^{17,18}$ and the association between hypertension and AF is also well recognized. ${ }^{19,20}$ While entirely speculative, one possible link between OSA and AF could be left atrial enlargement that occurs as a result of elevated left ventricular pressures from hypertension induced diastolic dysfunction. ${ }^{6,21}$ Diastolic dysfunction has been shown to be a powerful predictor of left atrium (LA) $\operatorname{size}^{22}$ and $\mathrm{AF}^{23}$ and several studies have demonstrated the independent association of diastolic dysfunction and OSA. ${ }^{24-26}$

Interatrial block (IAB) (defined as $P$-wave duration $\geq 120 \mathrm{~m}$ ) is recognized in the pathogenesis of AF. A study by our group demonstrated IAB was more prevalent in 144 patients with moderate-severe OSA (mean apnea hypopnea index $[\mathrm{AHI}]=56.2 \pm 27.9)$ compared to a control group of 36 patients with mild or no OSA (mean AHI $=5.6 \pm 3.6$ ) with incidences of $34.7 \%$ vs $0 \%$, respectively; $P<0.001$. The electrical remodeling leading to IAB may be mechanically mediated through intrathoracic pressure changes or a result of persistently elevated sympathetic tone..$^{21,27,28}$ This altered autonomic tone is also thought to have potential proarrhythmic effects on the PV ostia, as they are densely innervated by both vagal and adrenergic neurons. ${ }^{11}$

Overall, decreased parasympathetic activation predominates in severe OSA patients, however, there is evidence of rare increased parasympathetic activation toward the end of apneas in some. This is thought to occur as an oxygen conservation reflex in response to the apnea induced hypoxemia and is mediated through increased vagal tone. ${ }^{29}$ This reflex may produce significant bradycardia with a resultant reduction in the refractoriness of the atria, promoting an enhanced susceptibility to discharges from the PV ostia and thus AF. ${ }^{11}$

\section{Inflammation}

C-reactive protein (CRP) has been demonstrated as a sensitive marker for systemic inflammation and increased cardiovascular risk. ${ }^{30}$ It has also been noted to be elevated in some patients with OSA, though there have been some conflicting reports. A study by Shamsuzzaman et al demonstrated increased CRP in patients with OSA, and that the increase was proportional to the severity of OSA and independent of confounding disease states including obesity. ${ }^{31,32}$ A study by Chung et al demonstrated an association between elevated CRP and AF. ${ }^{33}$ They showed that CRP was $>2$-fold higher in AF patients when compared with controls, and that CRP was higher in the subgroup of patients with persistent compared to paroxysmal $\mathrm{AF}^{33}$ This suggests that there may be a possible association between OSA, inflammation, and AF, however, an explanation for this observation has yet to be determined. This link may be explained by the association of elevated CRP with volume overloaded states which in turn associates with both AF and OSA. ${ }^{34}$

\section{The association of AF and OSA}

Most observational studies, although not all, ${ }^{35,36}$ have suggested that cardiac arrhythmias are more frequent in OSA patients than in unaffected individuals ${ }^{37-40}$ and that the frequency of arrhythmias increases with the severity of apnea. ${ }^{38-40}$ One such study by Hoffstein et al ${ }^{38}$ prospectively followed 458 subjects undergoing polysomnography for evidence of cardiac arrhythmias and reported a $58 \%$ prevalence for those with OSA (AHI > 10) vs $42 \%$ in the controls $(\mathrm{AHI} \leq 10) ;(P<0.0001)$. They also demonstrated that the frequency of cardiac arrhythmias increased with the AHI, with $70 \%$ of patients with $\mathrm{AHI} \geq 40$ displaying arrhythmias vs $42 \%$ with an $\mathrm{AHI} \leq 10(P=0.002)$.

Unfortunately, many of the early studies cited above did not report AF separately from other forms of supraventricular arrhythmias which prevented clear associations with OSA from being derived. One of the first to clearly separate AF was Guilleminault et $\mathrm{al}^{37}$ who performed 24-hour Holter monitoring of 400 patients with moderate to severe OSA $(\mathrm{AHI} \geq 25)$ and reported a $3 \%$ prevalence of $\mathrm{AF}$ in the study, more than three times what is usually seen in a similar cohort from the general population of $0.4 \%-1 \%{ }^{11}$ Comparable results were also produced in the more recent Sleep Heart Health Study, a large cross-sectional study that assessed the prevalence of cardiac arrhythmias in 228 individuals with sleep-disordered breathing (SDB) (respiratory disturbance index $[\mathrm{RDI}]>30 /$ hour) and 338 without SDB (RDI $<5$ /hour). ${ }^{41}$ They demonstrated AF to be significantly more frequent in individuals with SDB than without, $4.8 \%$ vs $0.9 \%$ respectively, $P=0.003$. Notably, the 
authors did not differentiate central sleep apnea (CSA) from OSA which may have resulted in a slighter higher prevalence of AF in the SDB cohort than would have otherwise been seen. In general, the most common arrhythmias seen in the OSA population are: premature ventricular contractions (PVC), complex ventricular ectopy, nonsustained ventricular tachycardia, and AF. ${ }^{41}$

Despite some variability in the definition of OSA and of the SDB cohorts assessed, multiple studies utilizing several formats have shown a consistent association between OSA and AF, including a population-based study by Tanigawa et al that evaluated the frequency of oxygen desaturation and AF prevalence. ${ }^{42}$ They demonstrated that the odds ratio (OR) for AF was 2.47 (95\% cofidence interval [CI]: 0.91-6.69) for those subjects with 5-15 events/hour of 3\% oxygen desaturation index (ODI) and 5.66 (95\% CI: 1.75-18.34) for those with $\geq 15$ events/hour of $3 \%$ ODI $(P=0.020)$ even after adjustment for other confounders. This trend suggests that the association between OSA and AF prevalence is related to the extent of hypoxemia and therefore the severity of sleep apnea. Further evidence for this hypothesis was provided by Gami et al in a retrospective cohort study of 3,542 adults without prior history of AF who underwent polysomnography. ${ }^{29}$ They showed that for individuals $<65$ years old, both OSA (AHI $\geq 5$, hazard ratio [HR] 2.18, 95\% CI: 1.34-3.54) and a decrease in nocturnal oxygen saturation (per $0.5 \mathrm{U} \log$ change, HR 3.29, 95\% CI: 1.35-8.04) were independent predictors of incident AF. This study was also the first to demonstrate that for individuals aged $<65$ years, OSA and obesity were independent risk factors for incident AF.

Several studies have assessed the presence of OSA as a predictor of AF in specific subgroups including those post-cardiac surgery, post-electrical cardioversion, or with underlying $\mathrm{CHF},{ }^{43-45}$ Mooe et al performed polysomnography in 121 consecutive patients prior to coronary artery bypass grafting surgery to determine the relationship between OSA and the frequency of post-op AF requiring pharmacologic or electrical cardioversion. ${ }^{43}$ They showed that patients with an ODI $\geq 5$ had a significantly higher incidence of AF than those with an ODI $<5,39 \%$ vs $18 \%$ respectively, $P=0.02$. This resulted in a relative risk of AF of $2.8(95 \% \mathrm{CI}=1.2-6.8)$ for those with an ODI $\geq 5$ and this was independent of sex, hypertension, and ventricular function. A similar study by Kanagala et al evaluated the one year recurrence of AF postelectrical cardioversion. ${ }^{44}$ This study assessed 27 patients with untreated OSA (AHI $\geq 5$ ) and 79 control patients with an unknown OSA status. The rate of AF recurrence was significantly higher in the untreated group compared to the control group, $82 \%$ vs 53\% $(P=0.009)$. Statistical analysis demonstrated that AF recurrence was independent of BMI, age, and hypertension, but correlated with time spent at an $\mathrm{O}_{2}$ saturation $<90 \%$. That OSA predicts recurrence of AF post-cardioversion is not entirely unanticipated given that a significantly higher proportion of patients presenting for AF cardioversion have OSA compared to a general cardiology clinic population, $49 \%$ vs $32 \%$ respectively, $P=0.0004 .^{45}$

Heart failure patients are generally known to suffer from an increased burden of AF and thus the influence of sleep apnea in this population has been the focus of several studies. Javaheri et al investigated the association in a study of 81 subjects with stable CHF and ejection fraction $<45 \%$ who underwent polysomnography. ${ }^{46}$ They showed that $51 \%$ of the subjects enrolled had some form of SDB, with $40 \%$ having CSA and 11\% OSA. They also noted that subjects with SDB $(A H I \geq 15)$ had a significantly higher prevalence of AF than those without, $22 \%$ vs $5 \%$, respectively: $P=0.026$. Unfortunately, they did not report the distribution of OSA and CSA within the AF population. However, given the distribution of SDB it would be reasonable to assume that a significant proportion of those with AF had CSA. A study by $\mathrm{Sin}$ et al focused on a similar population of $\mathrm{CHF}$ patients to assess for SDB risk factors. ${ }^{47}$ They retrospectively studied 450 consecutive patients with CHF who were referred for polysomnography and showed that patients with CSA had a significantly higher prevalence of AF than those without, $23 \%$ vs $11.9 \%$, respectively; $P<0.05$. Of note, there was no significant difference in the prevalence of AF between OSA patients and those without SDB in the study, $11.9 \%$ and $7.5 \%$ respectively.

Whether SDB, in particular OSA, leads to AF is an appealing but currently unproven hypothesis. Controversy remains as to whether OSA is a primary etiologic factor for AF because of the high incidence of cardiovascular comorbidities in persons diagnosed with OSA. ${ }^{8}$ While there has been significant progress in separating individual risk factors for AF and OSA, they are complex disease processes with numerous dynamic interactions. This is highlighted by the ability to demonstrate the independence of AF risk between obesity and OSA only for individuals aged $<65$ years. ${ }^{29}$ One can only speculate, but it would seem plausible that aging dilutes the effect of OSA as other causes of AF begin to predominate such as hypertension, valvular heart disease, and diastolic dysfunction. A slightly different situation appears to occur in the CHF population where OSA is present but is less pervasive than CSA, suggesting that while 
it may be an important determinant of AF, in this setting it may be overshadowed. However, the exact roles of OSA and CSA in this population remain poorly defined as they are not well differentiated in many studies.

Also of importance are the few studies that have not shown a significant association between OSA and AF. One such casecontrol study by Porthan et al assessed the prevalence of OSA in 59 patients with lone AF and 56 matched controls. ${ }^{48}$ They showed that there was a high prevalence of OSA (AHI $\geq 15$ ) in the lone AF group but that it was not statistically different from the control group $32 \%$ vs $29 \%$, respectively; $P=0.67$. However, given the relatively high prevalence of OSA noted in the control group, some have suggested these discordant results may have been influenced by selection bias. ${ }^{11}$ Finally, as described above, there was no significant difference in the prevalence of $\mathrm{AF}$ in individuals with $\mathrm{CHF}$ regardless of OSA status as shown by $\mathrm{Sin}$ et $\mathrm{al}^{47}$ However, these results must be interpreted in caution as they are likely influenced by other etiologies of AF, most notably CSA.

\section{Effects of OSA therapy on AF}

While there is mounting evidence for a significant association between OSA and AF, the investigations that have been performed to date have tended to be small observational studies. These studies, while compelling, are subject to inherent flaws that prevent certainty of opinion. Of course, the role of OSA in the pathogenesis of cardiac arrhythmias would be greatly clarified if it could be shown that treatment of OSA altered the anticipated frequency of AF. Several studies have addressed this using a variety of interventional techniques including uvulopalatopharyngoplasty, tracheostomy, and continuous positive airway pressure (CPAP). ${ }^{37,49,50}$

Many early studies investigating OSA treatment and the prevalence of cardiac arrhythmias utilized tracheostomy to alleviate upper airway obstruction. Tilkian et al studied eight individuals with OSA for the presence of cardiac arrhythmias using several 24-hour Holter recordings pre- and post-tracheostomy. ${ }^{50}$ They noted a number of arrhythmias including second degree atrioventricular (AV) block, ventricular tachycardia, extreme sinus bradycardia and PVC, all of which reversed post-tracheostomy except PVCs. A similar but much larger study by Guillminault et al assessed 50 subjects with OSA (AHI $\geq 25)$ peri-tracheostomy for the presence of cardiac arrhythmias. ${ }^{37}$ They noted frequent episodes of sinus arrest, second degree AV block, PVCs, and extreme sinus bradycardia in several subjects as well as nocturnal paroxysmal AF in 10 of them. With the exception of frequent PVCs, all the aforementioned arrhythmias had resolved when reassessed 3-6 months post-tracheostomy.

There is mounting evidence to suggest that CPAP can reduce the frequency of $\mathrm{AF} .^{10} \mathrm{CPAP}$ is readily available, relatively simple to administer, and significantly less invasive than most alternative therapies for OSA and was thus utilized in a recent large observational intervention study. In this trial, Kanagala et al assessed the recurrence of AF after successful cardioversion and found that untreated OSA (AHI $\geq 5$ ) is associated with an $82 \%$ risk of recurrence vs $53 \%$ in the control group at one year, $(P=0.009) .{ }^{44}$ Interestingly, OSA patients who were effectively treated with CPAP were noted to have an even lower rate of AF recurrence than the control group $42 \%$ vs $53 \%$, suggesting that a portion of the controls may have suffered from undetected OSA, although the study did not compare them directly. Multivariate analysis showed that the risk of AF recurrence was related to the duration of nocturnal oxygen saturation $<90 \%$ and the magnitude of desaturation.

CPAP may reduce the frequency of AF by modifying some the inciting factors thought to promote AF in OSA including sympathetic activation, systemic inflammation, hypoxemia, cardiac dysfunction, and hypertension. While the preceding studies suggest that relief of airway obstruction and the resultant nocturnal desaturations in OSA patients with either tracheostomy or CPAP results in significant improvement or reversal of cardiac arrhythmias including AF, the current burden of evidence is insufficient to draw conclusive opinions, and will require further investigation with randomized trials to be resolved.

\section{Conclusions}

Most studies appear to suggest a significant association between OSA and an increased prevalence of cardiac arrhythmias, in particular AF. The pathophysiological connection between AF and OSA remains speculative, but appears to be the result of apnea induced hypoxia, intrathoracic pressure shifts, inflammation, heightened sympathetic activity, and autonomic instability leading to hypertension, diastolic dysfunction, LA enlargement, and electrical remodeling. Larger epidemiological studies are required to define the association while controlling for confounding conditions including CSA, obesity, and heart failure. While studies are limited, initial results appear promising that intervention with CPAP may be effective in reducing the burden of AF in this population, however, further investigations with randomized controlled trials will be necessary in order to provide certainty in this regard. 


\section{Disclosures}

The authors report no conflicts of interest in this work.

\section{References}

1. Fuster V, Ryden LE, Connom DS, et al. ACC/AHA/ESC 2006 guidelines for the management of patients with atrial fibrillation: a report of the American College of Cardiology/American Heart Association task force on practice guidelines and the European Society of Cardiology Committee for practice guidelines (writing committee to revise the 2001 guidelines for management of patients with atrial fibrillation): developed in collaboration with the European Heart Rhythm Association and the Heart Rhythm Society. Circulation. 2006;114: e257-e354.

2. Lloyd-Jones DM, Wang TJ, Leip EP, et al. Lifetime risk for development of atrial fibrillation: the Framingham heart study. Circulation. 2004;110:1042-1046.

3. Go AS, Hylek EM, Phillips KA, et al. Prevalence of diagnosed atrial fibrillation in adults: national implications for rhythm management and stroke prevention: the anticoagulation and risk factors in atrial fibrillation (ATRIA) study. JAMA. 2001;18:2370-2375.

4. Falk RH. Atrial fibrillation. N Engl J Med. 2001;344:1067-1067.

5. Benjamin EJ, Wolf PA, D'Agostino RB, et al. Impact of atrial fibrillation on the risk of death: the Framingham heart study. Circulation. 1998;98:946-952.

6. Baranchuk A, Simpson CS, Redfearn DP, Fitzpatrick M. It's time to wake up! Sleep apnea and cardiac arrhythmias. Europace. 2008;6:666-667.

7. Young T, Peppard PE, Gottleib DJ. Epidemiology of obstructive sleep apnea: a population health perspective. Am J Respir Crit Care Med. 2002; 165:1217-1239.

8. Somers VK, White DP, Amin R, et al. Sleep apnea and cardiovascular disease. An American Heart Association/American College of Cardiology Foundation scientific statement from the American Heart Association council for high blood pressure research professional education committee, council on clinical cardiology, stroke council, and council on cardiovascular nursing council. Circulation. 2008;118: 1080-1111.

9. Epstein LJ, Kristo D, Strollo PJ, et al. Clinical guideline for the evaluation, management, and long-term care of obstructive sleep apnea in adults. J Clin Sleep Med. 2009;3:263-276.

10. Gami AS, Friedman PA, Chung MK, et al. Therapy insight: interactions between atrial fibrillation and obstructive sleep apnea. Nat Clin Pract Cardiovasc Med. 2005;2:145-149.

11. Caples SM, Somers VK. Sleep-disordered breathing and atrial fibrillation. Prog Cardiovasc Dis. 2009;51:411-415.

12. Jais P, Haissaguerre M, Shah DC, et al. A focal source of atrial fibrillation treated by discrete radiofrequency ablation. Circulation. 1997;95:572-576.

13. Aydin M, Altin R, Ozeren A, Kart L, Bilge M, et al. Cardiac autonomic activity in obstructive sleep apnea. Tex Heart Inst J. 2004;31: $132-136$.

14. Narkiewicz K, Montano N, Cogliati C, et al. Altered cardiovascular variability in obstructive sleep apnea. Circulation. 1998;98:10711077.

15. Somers VK, Mark AL, Zavala DC, Abboud FM. Contrasting effects of hypoxia and hypercapnia on ventilation and sympathetic activity in humans. J Appl Physiol. 1989;67:2101-2106.

16. Somers VK, Dyken ME, Clary MP, Abboud FM. Sympathetic neural mechanisms in obstructive sleep apnea. J Clin Invest. 1995;96: 1897-1904.

17. Nieto FJ, Young TB, Lind BK, et al. Association of sleep-disordered breathing, sleep apnea, and hypertension in a large community-based study. JAMA. 2000;283:1829-1836.

18. Peppard PE, Young T, Palta M, Skatrud J. Prospective study of the association between sleep-disordered breathing and hypertension. N Engl J Med. 2000;342:1378-1384.
19. Kannel WB, Wolf PA, Benjamin EJ. Prevalence, incidence, prognosis, and predisposing conditions for atrial fibriallation: population-based estimates. Am J Cardiol. 1998;82:2N-9N.

20. Chugh SS, Blackshear JL, Shen WK, Hammill SC, Gersh BJ. Epidemiology and natural history of atrial fibrillation: clinical implications. J Am Coll Cardiol. 2001;37:371-378.

21. Parfrey B, Morriello F, Lim L, et al. Interatrial block in patients with sleep apnea [abstract]. Can J Cardiol. 2009;25 Suppl B:52B.

22. Pritchett AM, Mahoney DW, Jacobsen SJ, et al. Diastolic dysfunction and left atrial volume: a population-based study. J Am Coll Cardiol. 2005;45:87-92.

23. Tsang TS, Gersh BJ, Appleton CP, et al. Left ventricular diastolic dysfunction as a predictor of the first diagnosed nonvalvular atrial fibrillation in 840 elderly men and women. J Am Coll Cardiol. 2002;40:1636-1644.

24. Fung JW, Li TS, Choy DK, et al. Severe obstructive sleep apnea is associated with left ventricular diastolic dysfunction. Chest. 2002; 121:422-429.

25. Kraiczi H, Caidahl K, Samuelsson A, Peker Y, Hedner J. Impairment of vascular endothelial function and left ventricular filling: association with the severity of apnea-induced hypoxemia during sleep. Chest. 2001;119:1085-1091.

26. Alchanatis M, Paradellis G, Pini H, Tourkohoriti G, Jordanoglou J. Left ventricular function in patients with obstructive sleep apnea syndrome before and after treatment with nasal continuous positive airway pressure. Respiration. 2000;67:367-371.

27. Allessie M, Ausma J, Schotten U. Electrical, contractile, and structural remodeling during atrial fibrillation. Cardiovasc Res. 2002;54: 230-246.

28. Ariyarajah V, Spodick DH. The Bachmann bundle and interatrial conduction. Cardiol Rev. 2006;14:194-199.

29. Gami AS, Hodge DO, Herges RM, et al. Obstructive sleep apnea, obesity, and the risk of incident atrial fibrillation. J Am Coll Cardiol. 2007;49:565-571

30. Hatipoglu U, Rubinstein I. Inflammation and obstructive sleep apnea syndrome pathogenesis: a working hypothesis. Respiration. 2003;70:665-671.

31. Shamsuzzaman AS, Winnicki M, Lanfranchi P, et al. Elevated C-reactive protein in patients with obstructive sleep apnea. Circulation. 2002;105:2462-2464.

32. Punjabi NM, Beamer BA. C-reactive protein is associated with sleep disordered breathing independent of adiposity. Sleep. 2007;30: 29-34.

33. Chung MK, Martin DO, Sprecher D, et al. C-reactive protein elevation in patients with atrial arrhythmias: inflammatory mechanisms and persistence of atrial fibrillation. Circulation. 2001;104: 2886-2891.

34. Jo JPL, Lourenco P, Azevedo A, et al. Prognostic value of highsensitivity $\mathrm{C}$-reactive protein in heart failure: a systematic review. J Cardiac Fail. 2009; 15:256-266.

35. Flemons WW, Remmers JE, Gillis AM. Sleep apnea and cardiac arrhythmias. Is there a relationship? Am Rev Respir Dis. 1993;148: 618-621.

36. Koehler U, Schafer H. Is obstructive sleep apnea (OSA) a risk factor for myocardial infarction and cardiac arrhythmias in patients with coronary heart disease (CHD)? Sleep. 1996;19;283-286.

37. Guilleminault C, Connolly SJ, Winkle RA. Cardiac arrhythmia and conduction disturbances during sleep in 400 patients with sleep apnea syndrome. Am J Cardiol. 1983;52:490-494.

38. Hoffstein V, Mateika S. Cardiac arrhythmias, snoring, and sleep apnea. Chest. 1994;106:466-471.

39. Shepard JW, Garrison MW, Grither DA, Dolan GF. Relationship of ventricular ectopy to oxyhemoglobin desaturation in patients with obstructive sleep apnea. Chest. 1985;88:335-340.

40. Valencia-Flores M, Orea A, Castano VA, et al. Prevalence of sleep apnea and electrocardiographic disturbances in morbidly obese patients. Obes Res. 2000;8:262-269. 
41. Mehra R, Benjamin EJ, Shahar E, et al. Association of nocturnal arrhythmias with sleep-disordered breathing: the sleep heart health study. Am J Respir Crit Care Med. 2006;173:910-916.

42. Tanigawa T, Yanagishi K, Sakurai S, et al. Arterial oxygen desaturation during sleep and atrial fibrillation. Heart. 2006;92:1854-1855.

43. Mooe T, Gullsby S, Rabben T, Eriksson. Sleep-disordered breathing: a novel predictor of atrial fibrillation after coronary bypass surgery. Coron Artery Dis. 1996;7:475-478.

44. Kanagala R, Murali NS, Friedman PA, et al. Obstructive sleep apnea and the recurrence of atrial fibrillation. Circulation. 2003;107: 2589-2594.

45. Gami AS, Pressman G, Caples SM, et al. Association of atrial fibrillation and obstructive sleep apnea. Circulation. 2004;110:364-367.

46. Javaheri S, Parker TJ, Liming JD, et al. Sleep apnea in 81 ambulatory male patients with stable heart failure. Types and their prevalences, consequences, and presentations. Circulation. 1998;97: 2154-2159.
47. Sin DD, Fitzgerald F, Parker JD, et al. Risk factors for central and obstructive sleep apnea in 450 men and women with congestive heart failure. Am J Respir Crit Care Med. 1999;160:1101-1106.

48. Porthan KM, Melin JH, Kupila JT, et al. Prevalence of sleep apnea syndrome in lone atrial fibrillation: a case-control study. Chest. 2004;125:879-885.

49. Wright J, Johns R, Watt I, et al. Health effects of obstructive sleep apnea and the effectiveness of continuous positive airways pressure: a systematic review of the research evidence. BMJ. 1997;314:851-860.

50. Tilkian AC, Guilleminault C, Schroeder JS, et al. Sleep-induced apnea syndrome: prevalence of cardiac arrhythmias and their reversal after tracheostomy. Am J Med. 1977;63:348-358.

\section{Publish your work in this journal}

Nature and Science of Sleep is an international, peer-reviewed, open access journal covering all aspects of sleep science and sleep medicine, including the neurophysiology and functions of sleep, the genetics of sleep, sleep and society, biological rhythms, dreaming, sleep disorders and therapy, and strategies to optimize healthy sleep. The journal welcomes

\section{Dovepress}

original research, clinical \& epidemiological studies, reviews \& evaluations, case reports and extended reports. The manuscript management system is completely online and includes a very quick and fair peerreview system, which is all easy to use. Visit http://www.dovepress.com/ testimonials.php to read real quotes from published authors.

Submit your manuscript here: http://www.dovepress.com/nature-and-science-of-sleep-journal 